\title{
Variabilité ventilatoire et assistance ventilatoire en réanimation
}

\section{Breathing variability and mechanical ventilation in intensive care}

\author{
M. Schmidt • J. Cecchini $\cdot$ F. Kindler $\cdot$ T. Similowski $\cdot$ A. Demoule \\ Reçu le 15 octobre 2013 ; accepté le 18 décembre 2013 \\ (C) SRLF et Springer-Verlag France 2014
}

Résumé La ventilation n'est pas un phénomène monotone ; elle est au contraire variable dans le temps, non seulement au gré des ajustements homéostasiques aux besoins de l'organisme, mais aussi d'un cycle à l'autre (périodicité dite « anharmonique »). Cette variabilité cycle-à-cycle du volume courant et de ses composantes est liée à la nature complexe, pseudochaotique - au sens mathématique de ces termes - de la dynamique de la commande respiratoire centrale qui génère le débit ventilatoire. Une certaine variabilité respiratoire est synonyme de « bonne santé » respiratoire, et la diminution de la variabilité du comportement ventilatoire est pathologique. Cette diminution peut traduire soit des modifications centrales, soit le « filtrage » de la variabilité de la commande par des modifications de charge mécanique : la complexité du débit ventilatoire et la variabilité cycle-à-cycle de la ventilation sont liées au couplage neuromécanique respiratoire et à l'équilibre charge-capacité. Ainsi, en réanimation, une faible variabilité ventilatoire prédit l'échec du sevrage la ventilation mécanique. Elle est de plus un facteur indépendant de surmortalité. De plus, dans des modèles animaux, l'adjonction artificielle d'une variabilité extrinsèque améliore la mécanique respiratoire et les échanges gazeux. La restauration de la variabilité ventilatoire naturelle par certains modes d'assistance pourrait ainsi s'avérer bénéfique.

Mots clés Ventilation mécanique $\cdot$ Variabilité ventilatoire Complexité ventilatoire $\cdot$ Noisy ventilation

\footnotetext{
Abstract Ventilation is not monotonous. In contrast, the breathing pattern components vary with time, not only with respect to homeostatic adjustment needs, but also from one

M. Schmidt $(\bowtie) \cdot$ J. Cecchini $\cdot$ F. Kindler $\cdot$ T. Similowski $\cdot$

A. Demoule

Unité de réanimation et de surveillance continue, service de pneumologie et réanimation médicale, groupe hospitalier Pitié-Salpêtrière, Paris, France e-mail : matthieuschmidt@yahoo.fr

UMR-S 1158 Inserm-UPMC

« Neurophysiologie Respiratoire Expérimentale et Clinique »
}

cycle to another (often referred to as the "anharmonic" period). These breath-by-breath variations in tidal volume and its components are a result of the complex and chaotic nature - in mathematical terms - of the central ventilator command that creates the tidal volume. Respiratory variability reflects "healthy' breathing, whereas decreasing variability of the breathing pattern components is a reflection of "poor health". This decrease may be due to central command changes or, it may be due to the "filtering" of the central variability changes in regards to the mechanical loads: the complexity of the ventilatory flow and its breath-by-breath variability is related to both the neuro-mechanical coupling and the load-capacity adequacy. Thus, in intensive care, low ventilatory variability predicts mechanical ventilation weaning failure and is also an independent risk factor of death. Moreover, in animal models, the addition of extrinsic variability has shown improvements in both lung mechanics and gas exchange. The restoration of a natural variability through new mechanical ventilation modes may prove beneficial.

Keywords Mechanical ventilation - Ventilation variation Ventilation complexity $\cdot$ Noisy ventilation

\section{Introduction}

Au repos, la ventilation spontanée n'est pas monotone. Il est ainsi rare d'observer deux cycles respiratoires successifs en tout point superposables et identiques. Par conséquent, les grandeurs utilisées pour décrire le profil ventilatoire (volume courant $\left[\mathrm{V}_{\mathrm{T}}\right]$, fréquence ventilatoire $[\mathrm{FR}]$, temps inspiratoire [Ti] et expiratoire [Te], ventilation minute, etc.) varient dans le temps, spontanément ou à l'occasion de changements environnementaux. Cette variation est physiologique et semble témoigner d'un faible niveau de contrainte imposé à l'appareil respiratoire [1,2]. En revanche, la diminution de cette même variabilité est pathologique et témoigne d'une augmentation du niveau de charges imposées à l'appareil respiratoire [3-5]. Dans ce sens, les contraintes ventilatoires, quelle que soit leur nature, tendent à diminuer la variabilité 
du comportement ventilatoire. C'est ainsi que l'hypercapnie diminue la variabilité ventilatoire alors que l'hypocapnie l'augmente [6,7]. De la même façon, on observe une diminution de la variabilité ventilatoire lors de l'application, chez des sujets sains, de charges mécaniques résistives [4] ou élastiques [3]. Chez les patients atteints d'insuffisance respiratoire chronique restrictive, on observe également une diminution de la variabilité du comportement ventilatoire [5]. En réanimation, les contraintes imposées au système ventilatoire sont fréquentes; la ventilation mécanique, son sevrage et la sédation en sont trois exemples.

L'étude et la compréhension de ce qu'est réellement la variabilité ventilatoire en réanimation ont considérablement progressé au cours de ces dix dernières années. Initialement limitée au cadre d'une recherche qu'on pourrait qualifier de fondamentale, la variabilité ventilatoire trouve désormais des applications cliniques. Elle a ainsi un intérêt pronostique dans le sevrage de la ventilation mécanique et un intérêt thérapeutique dans le développement de nouveaux modes ventilatoires. Il s'agit typiquement d'un exemple de translation de données issues de la recherche fondamentale vers le lit du patient. L'objectif de la présente synthèse est de présenter les récentes avancées qui ont permis l'utilisation au lit du malade d'outils issus de l'étude de la variabilité ventilatoire en réanimation. Après un bref rappel des méthodes permettant l'analyse de la variabilité ventilatoire, nous détaillerons ses nouvelles applications au travers de nouveaux modes de ventilation assistée tels que la ventilation assistée proportionnelle $(\mathrm{PAV})^{1}[8,9]$, le neuro-asservissement de la ventilation assistée (NAVA) ${ }^{2}$ [10] ou encore la noisy ventilation ${ }^{\circledR 3}$ [11]. L'intérêt pronostique de la variabilité ventilatoire dans le sevrage ou dans la prédiction de la mortalité à court et long termes des patients de réanimation seront également présentés.

\section{Étude de la variabilité du comportement ventilatoire}

Les définitions et les moyens d'étude de la variabilité ventilatoire ayant déjà été largement détaillés dans un précèdent numéro de la revue Réanimation [12], nous n'aborderons ici que les prérequis nécessaires à la compréhension des plus récentes avancées en réanimation.

\section{Analyse de la variabilité cycle-à-cycle des variables ventilatoires}

En ventilation spontanée, le profil ventilatoire, défini par le $\mathrm{V}_{\mathrm{T}}$, le Ti, le Te, et la FR varie d'un cycle à l'autre. Cette variabilité, dite « cycle-à-cycle », décrite dès la fin $\mathrm{du} \mathrm{XIX}^{\mathrm{e}}$

1. Puritain Bennett 840, Covidien, Colorado, États-Unis

2. Servo-i, Maquet Critical Care, Solna, Suède

3. Evita XL 4, Dräger Medical AG, Lübeck, Germany siècle [13], est éminemment physiologique [1,2] et peut être très simplement appréhendée par le calcul du coefficient de variation qui se définit comme l'écart-type rapporté à la moyenne [14] (Tableau 1). Les valeurs «normales » du coefficient de variation des principaux descripteurs du profil ventilatoire ne sont pas formellement établies. Néanmoins, les valeurs rapportées par Tobin et al. chez 65 volontaires sains, après 15 minutes de ventilation spontanée, peuvent servir de référence $[2,15]$. Le coefficient de variation du $\mathrm{V}_{\mathrm{T}}$, de la $\mathrm{FR}$ et du Ti rapporté à la durée totale du cycle (Ti/Ttot) étaient respectivement de $33,0 \pm 14,9 \%, 20,8 \pm 11,5 \%$, et $17,7 \pm$ $6,5 \%$. Cette même variabilité peut aussi être décrite par la force de la relation qui existe entre un cycle ventilatoire et le suivant, autrement appelée autocorrélation (concept de " mémoire à court terme ») [14]. Ainsi, l'autocorrélation peut être décrite par le « $\mathrm{r}-\mathrm{lag}^{-1} »$ qui correspond au coefficient d'autocorrélation entre un cycle et le suivant ou encore par le « lag » défini par le nombre de cycles respiratoires séparant deux cycles significativement corrélés. Plus complexe, l'analyse spectrale par transformée de Fourier permet de décomposer le profil ventilatoire selon ses pics de fréquence en trois fractions : périodique, autocorrélée et aléatoire [16]. Cette approche, qui a fait très largement ses preuves dans le domaine de la variabilité cardiaque, semble en pratique efficace pour décrire la variabilité ventilatoire.

\section{Analyse de la complexité de la commande ventilatoire et de sa traduction en débit}

La variabilité cycle-à-cycle du comportement ventilatoire provient du fait que la commande ventilatoire centrale n'est pas produite par des oscillateurs « sinusoïdaux », mais par des oscillateurs dits « complexes » qui produisent un signal de sortie " anharmonique ». On utilise le terme « système complexe » pour désigner des ensembles constitués d'un grand nombre d'entités en interaction, dont le comportement ou l'évolution est imprévisible, tout en restant inscrit dans une gamme prédictible. Ainsi, si l'on représente l'évolution d'un signal physiologique cyclique et « complexe » au cours du temps dans un espace tridimensionnel, on constate que chaque « cycle » est représenté par une figure irrégulière qui ne recouvre pas la précédente et n'est pas recouverte par la suivante (Fig. 1). Au contraire, le « portrait de phase à trois dimensions » d'un signal cyclique « linéaire », ou « périodique» est simple et « monotone » (Fig. 1). On peut décrire la complexité d'un signal au moyen d'outils mathématiques non linéaires pour la plupart dérivés de la théorie du chaos (contrairement à son usage habituel, le terme chaos ne fait pas ici référence au désordre ou à la confusion, mais à une théorie mathématique dont l'application pratique la plus « populaire » est la prévision météorologique). Très schématiquement, on parle de « chaos » pour décrire une dynamique périodique anharmonique dans un système déterministe 
Tableau 1 Principaux outils d'analyse de la variabilité ventilatoire

\begin{tabular}{|c|c|}
\hline Types d'analyses & Définitions \\
\hline \multicolumn{2}{|l|}{ Variabilité ventilatoire cycle à cycle (analyse linéaire) } \\
\hline Coefficient de variation & Écart-type rapporté à la moyenne [14] \\
\hline Lag & $\begin{array}{l}\text { Autocorrélation : nombre de cycle respiratoire significativement } \\
\text { corrélés les uns aux autres [14] }\end{array}$ \\
\hline R-lag ${ }^{-1}$ & Coefficient d'autocorrélation entre le premier et le second cycle [14] \\
\hline Analyse spectrale par transformé de Fourier & Décompose le profil ventilatoire selon leur pics de fréquence [16] \\
\hline \multicolumn{2}{|l|}{ Complexité ventilatoire (analyse non linéaire) } \\
\hline $\begin{array}{l}\text { Méthode de titration de bruit ou « noise titration », } \\
\text { déterminant une quantité de bruit titrante ou } \\
\text { noise limit (NL) }\end{array}$ & $\begin{array}{l}\text { Quantité de « bruit blanc » nécessaire à masquer la composante non } \\
\text { linéaire de la variabilité. Une } \mathrm{NL}>0 \text { témoigne de la présence } \\
\text { de complexité et la quantifie [21] }\end{array}$ \\
\hline $\begin{array}{l}\text { Le plus grand des exposants de Lyapunov (LLE) : } \\
\text { mesure de la sensibilité aux conditions initiales }\end{array}$ & $\begin{array}{l}\text { Mesure à quel point une perturbation dans le passé peut affecter } \\
\text { le comportement futur d'un système. Plus le LLE est grand, plus } \\
\text { le système est sensible aux conditions initiales [25] }\end{array}$ \\
\hline $\begin{array}{l}\text { Entropie de Kolmogorov-Sinaï (KSE) : mesure } \\
\text { de l'imprédictibilité d'un système complexe }\end{array}$ & $\begin{array}{l}\text { Estimée par la somme des LLE positifs. Une faible KSE témoigne } \\
\text { d'un système prédictif et régulier, à l'inverse d'une valeur élevée qui } \\
\text { reflète un système imprédictible et fortement variable [25] }\end{array}$ \\
\hline Portrait de phase en trois dimensions & Représentation graphique de la nature du système \\
\hline
\end{tabular}

\section{Système périodique}

\section{Système chaotique (complexe)}
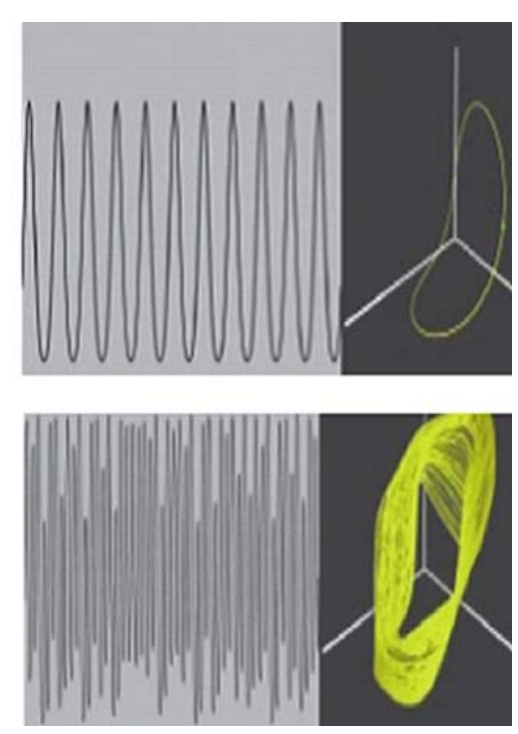

Fig. 1 Représentation du portrait de phase à trois dimensions d'un signal « périodique » et d'un signal physiologique « complexe»

sensible aux conditions initiales [17]. Des débats animés opposent physiciens et mathématiciens sur la définition du chaos et la façon de le mettre en évidence. Pour certains, qualifier de « chaotique » la ventilation des mammifères est impropre, parce qu'il n'est pas possible de prouver mathématiquement un "déterminisme » (un «but», une « orientation » en langage simple) qui est pourtant physiologiquement évident (« assurer la vie et l'homéostasie »). Il faudrait en toute rigueur employer des termes comme «pseudo-chaotique », ou « chaos compatible »; en pratique, parler de complexité suffit à exprimer le message souhaité.
De nombreuses données scientifiques démontrent le caractère complexe de la ventilation $[18,19]$. Chez l'humain, le sommeil lent profond, qui supprime les influences afférentes et corticales qui s'exercent sur le contrôle ventilatoire, est associé à une diminution marquée de la complexité ventilatoire [20], ce qui est aussi le cas de l'hypocapnie [6].

L'automatisation des calculs (Matlab, Mathworks, Natick, MA, États-Unis ; Dataplore, Datan, Teltow, Germany) et le développement de nouvelles méthodes d'analyse ont rendu plus accessible l'étude non linéaire de la variabilité ventilatoire. Une méthode récente, dite « titration de bruit» (noise titration en anglais donnant accès à la noise limit) [21] donne accès à une évaluation quantitative de la complexité ventilatoire, et permet, si certaines précautions méthodologiques sont respectées, de caractériser d'éventuels changements d'état au cours du temps (par exemple en réponse à l'administration d'un bronchodilatateur au cours de la bronchopneumopathie chronique obstructive [22] ou en réponse à la modification d'une assistance ventilatoire [23,24] (Tableau 1). Brièvement, cette technique recherche la présence d'une composante non linéaire de la variabilité puis mesure la quantité de « bruit blanc » nécessaire à la masquer. Un peu comme la titration d'un acide par une base. Une noise limit supérieure à zéro témoigne du caractère non linéaire du signal traité (par exemple débit ventilatoire) et sa valeur est proportionnelle au degré de complexité. Une fois affirmée la complexité du signal, on peut en décrire d'autres caractéristiques, comme par exemple la sensibilité aux conditions initiales. En d'autres termes, il s'agit d'évaluer à quel point une perturbation dans le passé peut affecter le comportement futur d'un système. La sensibilité aux conditions initiales peut être décrite par le 
calcul du plus grand des exposants de Lyapunov (LLE) [25]. Plus le LLE est élevé, plus le système est sensible aux conditions initiales. Une autre caractéristique d'un système complexe est son imprédictibilité. Celle-ci est estimée par la somme des exposants de Lyapunov positifs, autrement appelée entropie de Kolmogorov-Sinai (KSE) [26]. Ainsi une faible KSE témoigne d'un système prédictif et régulier, à l'inverse un KSE élevé reflète un système imprédictible et fortement variable. Enfin, cette complexité peut être représentée par le portrait de phase en trois dimensions, représentation graphique de la nature du système (voir ci-dessus, et Fig. 1).

Chez les patients de réanimation, l'échec du sevrage de la ventilation mécanique semble être associé à une complexité supérieure du $V_{T}$ [27]. Par ailleurs, alors que les patients ventilés de façon contrôlée ne présentent majoritairement pas de complexité ventilatoire, le passage en ventilation spontanée avec aide inspiratoire (VSAI) s'accompagne chez les mêmes patients de l'apparition d'une telle complexité [28]. Ceci suggère donc fortement que la complexité ventilatoire est fortement liée à l'activité des centres de la commande ventilatoire, isolés ou sous l'influence des afférences d'origine respiratoire [6]. Ceci implique de souligner l'importance de la nature du signal étudié pour interpréter la complexité ventilatoire et son évolution au cours du temps. Ainsi, plus le signal étudié est « en aval» de la commande ventilatoire, moins l'information fournie porte-t-elle « purement» sur celle-ci. Ce concept est bien illustré par une étude des évolutions concomitantes de la complexité ventilatoire du signal électromyographique du diaphragme (très « proche » de la commande ventilatoire) d'une part, et du débit ventilatoire (commande ventilatoire « filtrée » par les propriétés mécaniques respiratoires) d'autre part, au cours d'une augmentation progressive de l'assistance ventilatoire chez des patients de réanimation [24]. Dans cette étude, l'assistance ventilatoire influait peu sur la complexité " centrale », mais avait un effet spectaculaire sur la complexité « périphérique » (Fig. 2). La complexité du débit ventilatoire était donc dans ce cas plus descriptive du couplage neuromécanique (équilibre charge-capacité) que de la commande centrale.

Au terme de ce qui précède, l'analyse de la variabilité du comportement ventilatoire trouve donc naturellement sa place dans l'étude des interactions patient-ventilateur.

\section{Variabilité et modes proportionnels : un intérêt thérapeutique}

\section{Principe de fonctionnement des modes proportionnels}

Les modes ventilatoires partiels regroupent l'ensemble des modes ventilatoires autorisant une participation du patient à l'effort ventilatoire, que ce soit pour le déclenchement du ventilateur ou sa contribution à la genèse du volume courant.

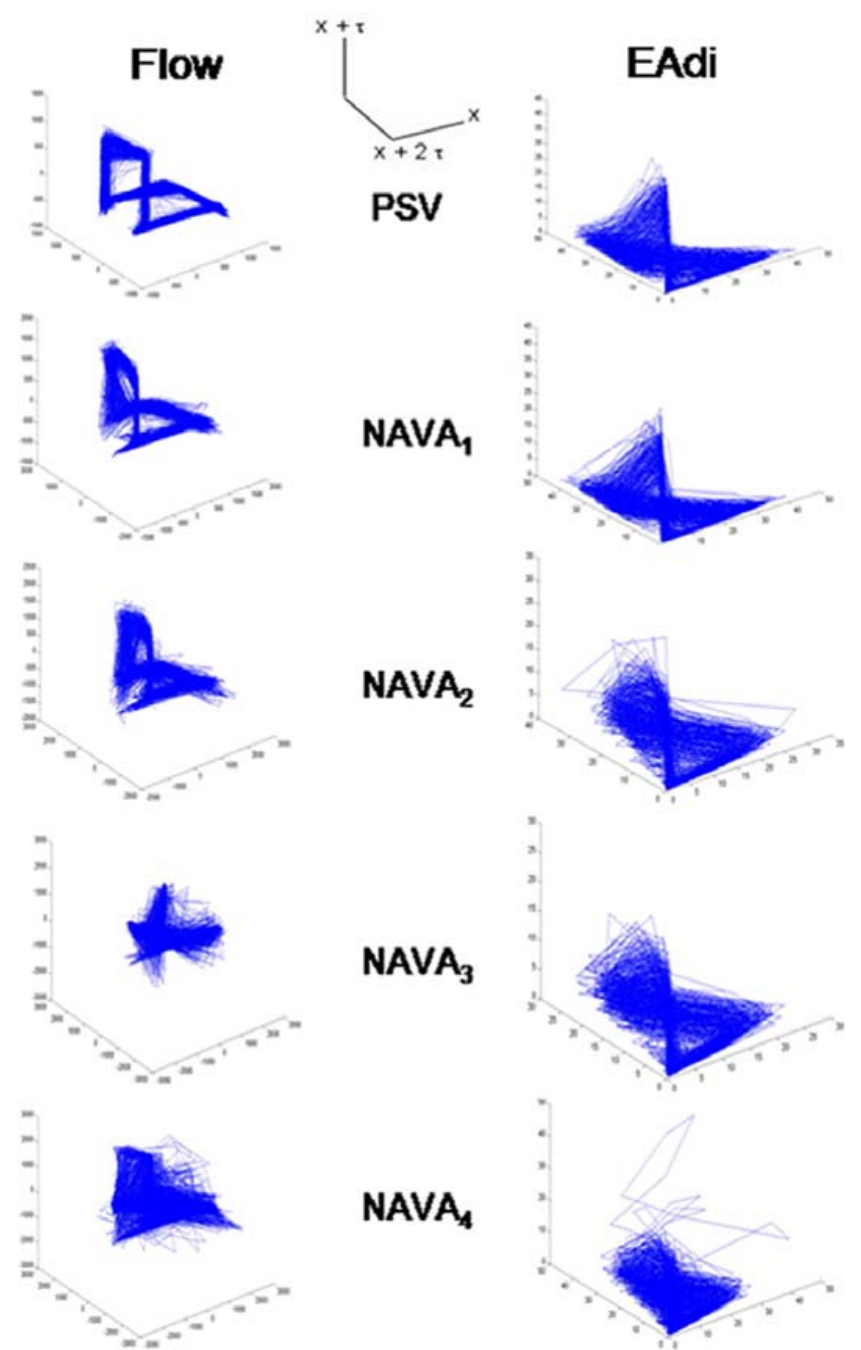

Fig. 2 Portrait de phase en trois dimensions du débit (A) et du l'activité électrique du diaphragme (EAdi) en ventilation spontané avec aide inspiratoire (PSV) et a 4 niveaux niveau de NAVA de 1 à 4 cmH2O/ $\mu \mathrm{V}$ d'EAdi (NAVA1, 2, 3, 4). D'après [24]. La complexité ventilatoire, du débit (A) était supérieure en mode NAVA et augmentait parallèlement au niveau de NAVA tandis que la complexité de l'EAdi restait constante. Ces résultats suggéraient que le mode NAVA n'influençait de fait pas la commande centrale, mais l'adéquation charge-capacité ou couplage neuro-mécanique. La complexité $\mathrm{du}$ débit ventilatoire était donc dans ce cas plus descriptive du couplage neuro-mécanique (équilibre charge-capacité) que de la commande centrale. Ce mode pourrait permettre de révéler l'expression de la variabilité et de la complexité de la commande ventilatoire centrale des patients ventilés. EAdi : activité électrique du diaphragme ; NAVA : neuro-asservissement de la ventilation assistée ; VSAI : ventilation spontanée avec aide inspiratoire

Ainsi, en ventilation partielle, la pression musculaire développée par le patient n'est pas nulle, contrairement aux modes contrôlés, où seuls le ventilateur et les réglages qui lui sont attribués participent à la gestion de l'ensemble des 
éléments du cycle ventilatoire. Selon les rapports entre la pression d'assistance délivrée par le ventilateur $\left(\mathrm{P}_{\text {vent }}\right)$ et la pression musculaire fournie par le patient $\left(\mathrm{P}_{\mathrm{mus}}\right)$, deux groupes d'assistance partielle peuvent être distingués :

- le premier est représenté par la VSAI. Ici, la pression d'assistance reste constante quelle que soit la pression musculaire développée par le patient. Quand le patient augmente son effort musculaire, le $\mathrm{V}_{\mathrm{T}}$ augmente, mais avec un rendement qui reste constant, identique à celui du patient sans assistance ;

- le second regroupe les modes reposant sur la proportionnalité de l'assistance à l'effort inspiratoire du patient. Dans ces modes dits " proportionnels », l'assistance délivrée par le ventilateur augmente à mesure que la demande ventilatoire du patient augmente, et réciproquement. De ce fait, la pression d'assistance délivrée par le ventilateur devient variable, adaptée aux variations de demande ventilatoire survenant au cours du temps, à la fois entre chaque cycle et au sein même d'un cycle respiratoire. Les modes PAV (Puritain Bennett 840, Covidien, Colorado, USA) et NAVA (Servo-i, Maquet Critical Care, Solna, Suède) en sont les deux principaux représentants actuels.

En agissant au plus proche de la commande ventilatoire du malade, ces modes proportionnels s'affranchissent d'un des principaux écueils des modes dits « conventionnels »e.g. la VSAI - qui est d'admettre à tort que la demande ventilatoire reste stable au cours du temps et de délivrer une assistance fixe. Pour ce faire, la PAV et le NAVA vont fonctionner comme des amplificateurs de la commande ventilatoire en délivrant une pression proportionnelle à l'activité musculaire inspiratoire en PAV ou à l'activité électrique du diaphragme (EAdi) en NAVA.

\section{Ventilation assistée proportionnelle et variabilité}

Lors de la PAV, le ventilateur délivre une assistance en pression proportionnelle à l'effort musculaire instantané du patient. Cette proportionnalité s'applique de façon continue au cours de l'inspiration $[8,9]$ et nécessite de connaître l'effort fourni par le patient ainsi que la mécanique de son système respiratoire (compliance et résistance). Cet effort, la compliance et la résistance ainsi sont mesurés de façon très rapprochée au moyen d'algorithmes complexes En pratique, pour le clinicien, le réglage du ventilateur s'effectue sur un gain représentant le pourcentage de la pression totale appliquée pris en charge par le ventilateur.

Chez 13 patients mécaniquement ventilés successivement en PAV et en VSAI dans des conditions d'assistance comparables (pression inspiratoire moyenne similaire dans les deux conditions), le $\mathrm{V}_{\mathrm{T}}$ moyen était identique dans les deux conditions tandis que sa variabilité était significativement supérieure en PAV ( $10 \pm 4 \%$ vs $20 \pm 13 \%$ ) [29]. De plus, la baisse du niveau d'assistance en PAV (diminution de la proportion de « l'effort inspiratoire » prise en charge par le ventilateur), affectait peu la variabilité du $V_{T}$ qui restait supérieure en PAV par rapport au mode VSAI [29]. Ainsi, en s'adaptant à l'activité musculaire respiratoire propre du patient sans lui imposer une assistance fixe comme le fait la VSAI, le mode PAV permet de restaurer la variabilité du comportement ventilatoire [26-30]. Plusieurs études comparant les modes PAV et VSAI en ventilation non invasive dans une population générale de patients de réanimation $[30,31]$ ou spécifiquement chez des patients ayant une broncho-pneumopathie chronique obstructive $[32,33]$ ont démontré, en se basant sur une échelle visuelle analogique, une amélioration du confort respiratoire en PAV. Un meilleur respect de la variabilité ventilatoire en PAV pourrait en être à l'origine. De même, le mode PAV améliore l'interaction patient-ventilateur par une réduction de la $\mathrm{P}$ 0.1 [34], des appels inefficaces $[35,36]$ et plus largement des asynchronies patients ventilateurs [37].

\section{Neuro-asservissement de la ventilation assistée et variabilité}

Alors que la PAV délivre une assistance proportionnelle à l'effort musculaire inspiratoire, le NAVA délivre quant à lui une assistance ventilatoire proportionnelle à EAdi, témoin direct de l'activité de la commande ventilatoire centrale [38]. Ce rapprochement de la commande centrale se traduit par une augmentation de la variabilité ventilatoire. Dans trois études récentes où le débit ventilatoire et l'EAdi étaient recueillis en VSAI puis en NAVA le coefficient de variation des descripteurs du cycle ventilatoire était supérieur en mode NAVA comparé à la VSAI [24,39,40]. De plus, elle augmentait avec le niveau de NAVA, déterminant du niveau d'assistance [24]. La représentation graphique de cette variabilité est présentée dans la Figure 3. La restauration de cette variabilité par la NAVA ne se limitait pas au $\mathrm{V}_{\mathrm{T}}$ mais concernait l'ensemble des descripteurs du cycle ventilatoire $\left(\mathrm{V}_{\mathrm{T}}, \mathrm{FR}\right.$, pression maximale et pression moyenne) [39]. En revanche, une augmentation trop importante de la variabilité à des hauts niveaux de NAVA (i.e $>5 \mathrm{cmH}_{2} \mathrm{O} / \mu \mathrm{V}$ ) pouvait être associée, chez certains patients, à des volumes courants supérieurs à $10 \mathrm{~mL} /$ $\mathrm{kg}$ et à un inconfort [40]. La complexité ventilatoire, évaluée par le LLE du débit était elle aussi supérieure en mode NAVA et augmentait parallèlement au niveau de NAVA tandis que le LLE de l'EAdi restait constant [24]. Ces résultats suggéraient que le mode NAVA n'influençait de fait pas la commande centrale, mais l'adéquation charge-capacité ou couplage neuro-mécanique (Figure 2). Ce mode pourrait permettre de révéler l'expression de la variabilité et de la complexité de la commande ventilatoire centrale des patients ventilés [24].

Malgré l'avancée des connaissances et l'engouement pour ces modes ventilatoires proportionnels (PAV et NAVA), l'impact clinique et le bénéfice à long terme de la 

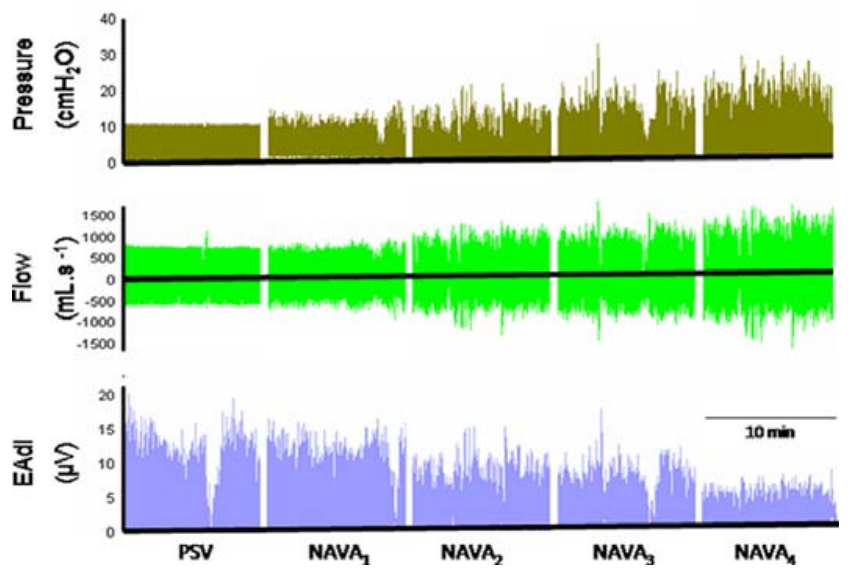

Fig. 3 Variabilité du profil ventilatoire en VSAI et après une augmentation du niveau de NAVA de 1 à $4 \mathrm{cmH} 2 \mathrm{O} / \mu \mathrm{V}$ d'EAdi (NAVA1, 2, 3, 4). D'après [24]. EAdi : activité électrique du diaphragme ; NAVA : neuro-asservissement de la ventilation assistée ; VSAI : ventilation spontanée avec aide inspiratoire

restauration de la variabilité ventilatoire par ces modes restent mal définis et peu étudiés.

\section{Un mode ventilatoire basé sur la variabilité : la noisy ventilation}

Une des nouveautés dans le domaine de la variabilité ventilatoire en réanimation a été le développement d'un mode ventilatoire qui intègre la variabilité dans son algorithme avec pour objectif de délivrer un volume courant variable [41]. Ce mode qui fait varier le volume courant se nomme noisy ventilation ${ }^{\circledR}$ ou Ventilation Variable (Evita XL 4, Dräger Medical AG, Lübeck, Germany). Il permet de « mimer » la variabilité « naturelle » du comportement ventilatoire [42]. Bien que les études humaines fassent défaut à ce jour (octobre 2013), la noisy ventilation ${ }^{\circledR}$ montre des résultats intéressants, que ce soit en ventilation assistée $[43,44]$ ou contrôlée $[45,46]$.

En effet, plusieurs études animales ont montré que la noisy ventilation $^{\circledR}$ améliorait les échanges gazeux [47-49], diminuait la réponse inflammatoire [50] et réduisait le dommage alvéolaire [51]. Dans un travail récent ayant comparé la noisy ventilation $^{\circledR}$ en ventilation assistée (e.g. VSAI avec addition de « bruit ») à la VSAI dans un modèle porcin d'insuffisance respiratoire aiguë, la noisy ventilation ${ }^{\circledR}$ était associée à un coefficient de variation du $\mathrm{V}_{\mathrm{T}}$ plus élevé, une $\mathrm{PaCO}_{2}$ et des efforts inspiratoires plus faibles, et une diminution de l'œdème pulmonaire et de l'inflammation alvéolaire dans les zones non ventilées [11]. De façon similaire, si la variabilité est appliquée en pression contrôlée, la noisy ventilation ${ }^{\circledR}$ semblait également réduire les phénomènes d'ouverturefermeture alvéolaire et de distension dynamique. De même, la noisy ventilation ${ }^{\circledR}$ pourrait permettre une meilleur redistri- bution dorso-ventrale et cranio-caudale de la perfusion pulmonaire $[11,43,52]$. Plusieurs mécanismes pourraient expliquer l'amélioration des échanges gazeux et de la mécanique ventilatoire avec ce nouveau mode : le phénomène de résonance stochastique, qui consiste à amplifier un signal par l'addition de « bruit » [41], l'augmentation du surfactant endogène [53], un meilleur recrutement alvéolaire [54], un meilleur rapport ventilation/perfusion [43], voire un désécrantage alvéolaire [55]. La noisy ventilation ${ }^{\circledR}$ semble donc avoir des bénéfices potentiels en pratique clinique. De larges études humaines sont néanmoins nécessaires pour confirmer la transposition de ces données animales à l'humain. Comparé aux modes proportionnels (NAVA et PAV) un des atouts de la ventilation variable pourrait être sa simplicité d'utilisation et de fonctionnement pour le clinicien. En effet, comparé à la VSAI, le seul réglage nouveau est la proportion de variabilité que le clinicien souhaite introduire. Très récemment, la noisy ventilation $^{\circledR}$, la PAV et la VSAI ont été comparées dans un modèle porcin d'insuffisance respiratoire aiguë. La noisy ventilation $^{\circledR}$ et la PAV permettaient toutes deux d'obtenir une meilleure oxygénation et une plus grande variabilité du $V_{T}$ par rapport à la VSAI. En revanche, l'inflammation pulmonaire et le dommage alvéolaire histologique étaient similaires dans les trois groupes [44].

L'introduction d'un certain niveau de la variabilité ventilatoire extrinsèque, (i.e. noisy ventilation ${ }^{\circledR}$ ) ou la restauration de la variabilité intrinsèque du comportement ventilatoire (i.e. PAV et NAVA) offre donc des résultats prometteurs. En revanche, son impact sur le pronostique en réanimation doit être maintenant démontré [56].

\section{La variabilité ventilatoire : un intérêt pronostique}

\section{Variabilité ventilatoire et sevrage}

Le sevrage est une situation caractéristique où le système respiratoire doit faire face à une somme de charges mécaniques ou métaboliques. Le succès de cette épreuve est principalement déterminé par la nature du rapport entre les charges imposées au système respiratoire et la capacité des muscles ventilatoires à les surmonter. L'utilisation des indices de variabilité dans le but de prédire le succès du sevrage de la ventilation mécanique a été largement étudiée. Il est ainsi montré que les malades extubés avec succès ont une variabilité ventilatoire supérieure à celle des patients en échec d'extubation [57-59]. Un coefficient de variation du rapport $\mathrm{V}_{\mathrm{T}} / \mathrm{Ti} \geq 19 \%$ et du rapport $\mathrm{Ti} / \mathrm{Ttot} \geq 10 \%$ après une épreuve de ventilation spontanée sur pièce en $\mathrm{T}$ de 60 minutes prédit assez précisément le succès du sevrage [59]. De plus, la compliance dynamique était significativement corrélée au coefficient de variation $\mathrm{du}$ rapport $\mathrm{Ti} / \mathrm{Ttot}(\mathrm{R}=0.605$ 
[0,285-0,804], $\mathrm{p}=0,0005)$ [59]. Il faut néanmoins souligner que ces résultats reposaient sur l'étude de la variabilité ventilatoire et non pas de la complexité dont les résultats sont plus discordants dans le sevrage [27,60]. De même, les conditions de l'épreuve de sevrage influencent la variabilité ventilatoire [58]. Comparée à l'application d'une pression expiratoire positive (PEP) de $5 \mathrm{cmH}_{2} \mathrm{O}$ seule et à l'association d'une aide inspiratoire et d'une $\mathrm{PEP}$, toutes deux réglées à $5 \mathrm{cmH}_{2} \mathrm{O}$, l'analyse de la variabilité des composants du comportement ventilatoire au cours d'une pièce en $\mathrm{T}$ était la plus performante pour prédire le succès du sevrage (aire sous la courbe entre 0,73 et 0,87 ) [58].

\section{Variabilité ventilatoire et mortalité en réanimation}

Au-delà du sevrage de la ventilation mécanique de très récentes données suggèrent que la variabilité ventilatoire pourrait être un facteur pronostique de mortalité en réanimation. Une étude vient ainsi de montrer que chez 178 patients ventilés en réanimation dont le profil ventilatoire a été enregistré de façon continue, la mortalité à 28 et 180 jours était significativement supérieure chez les patients ayant une variabilité de la FR $<40 \%$. En analyse multivariée, une faible variabilité de la FR était un facteur de risque indépendant de mortalité à 28 jours [61]. Cependant, la sédation, fréquente en ventilation mécanique, pourrait avoir influencé ces résultats. En effet, il est montré que l'arrêt de la sédation en ventilation mécanique est associé à une ascension de la variabilité de la fréquence cardiaque, ce quelle que soit la sévérité du patient. En revanche, l'augmentation de la variabilité de la fréquence respiratoire n'est observée que chez les patients ayant un faible score de défaillance d'organe, suggérant un lien étroit entre la diminution de la variabilité respiratoire et la sévérité du patient, ce au-delà du niveau de sédation [62].

\section{Conclusion}

La description de la variabilité ventilatoire en réanimation et la compréhension de ses mécanismes sous-jacents ont beaucoup progressé au cours de la dernière décennie. La variabilité ventilatoire n'a plus uniquement un intérêt pronostique. En effet, les résultats prometteurs des modes proportionnels et de la noisy ventilation ${ }^{\circledR}$, tous associés à un niveau de variabilité supérieur à celle des modes plus conventionnels nous montrent que la variabilité pourrait devenir un outil thérapeutique en ventilation mécanique. L'impact clinique d'une ventilation plus variable, aux travers de ces nouveaux modes ventilatoires, doit maintenant être évalué par des essais cliniques.

Conflit d'intérêt : L'Association pour le Développement et l'Organisation de la Recherche en Pneumologie (ADOREP) a reçu le soutien financier de Maquet SA, Orléans pour la conduite de travaux de recherche sur la NAVA.

\section{Références}

1. Dejours P, Bechtel-Labrousse Y, Monzein P, Raynaud J (1961) [Study of the diversity of ventilatory rates in man]. J Physiol (Paris) 53:320-1

2. Tobin MJ, Mador MJ, Guenther SM, et al (1988) Variability of resting respiratory drive and timing in healthy subjects. J Appl Physiol (1985) 65:309-17

3. Brack T, Jubran A, Tobin MJ (1997) Effect of elastic loading on variational activity of breathing. Am J Respir Crit Care Med $155: 1341-8$

4. Brack T, Jubran A, Tobin MJ (1998) Effect of resistive loading on variational activity of breathing. Am J Respir Crit Care Med 157:1756-63

5. Brack T, Jubran A, Tobin MJ (2002) Dyspnea and decreased variability of breathing in patients with restrictive lung disease. Am J Respir Crit Care Med 165:1260-4

6. Fiamma MN, Straus C, Thibault S, et al (2007) Effects of hypercapnia and hypocapnia on ventilatory variability and the chaotic dynamics of ventilatory flow in humans. Am J Physiol Regul Integr Comp Physiol 292:R1985-1993.

7. Jubran A, Grant BJ, Tobin MJ (1997) Effect of hyperoxic hypercapnia on variational activity of breathing. Am J Respir Crit Care Med 156(4 Pt 1):1129-39

8. Younes M, Puddy A, Roberts D, et al (1992) Proportional assist ventilation. Results of an initial clinical trial. Am Rev Respir Dis 145:121-9

9. Younes M (1992) Proportional assist ventilation, a new approach to ventilatory support. Theory. Am Rev Respir Dis 145:114-20

10. Sinderby C, Navalesi P, Beck J, et al (1999) Neural control of mechanical ventilation in respiratory failure. Nature medicine 5:1433-6

11. Gama de Abreu M, Spieth PM, Pelosi P, et al (2008) Noisy pressure support ventilation: a pilot study on a new assisted ventilation mode in experimental lung injury. Crit Care Med 36:818-27

12. Wysocki MCC, Teixeira A, Strauss C, et al (2006) La variabilité du comportement ventilatoire : perspectives pour le malade de réanimation. Réanimation 15:7-13

13. Marey J (1864) Etudes graphiques des mouvements respiratoires. CR Soc Biol 1:175-81

14. Benchetrit G, Bertrand F (1975) A short-term memory in the respiratory centres: statistical analysis. Respir Physiol 23:147-58

15. Tobin MJ, Chadha TS, Jenouri G, et al (1983) Breathing patterns. 1. Normal subjects. Chest $84: 202-5$

16. Modarreszadeh M, Bruce EN, Gothe B (1990) Nonrandom variability in respiratory cycle parameters of humans during stage 2 sleep. J Appl Physiol (1985) 69:630-9

17. Kaplan D, Glass L (1995) Finite-difference equations. Understanding nonlinear dynamics. New-York: Springer-Verlag

18. Donaldson GC (1992) The chaotic behaviour of resting human respiration. Respir Physiol 88:313-21

19. Wysocki M, Fiamma MN, Straus C, et al (2006) Chaotic dynamics of resting ventilatory flow in humans assessed through noise titration. Respir Physiol Neurobiol 153:54-65

20. Sako T, Burioka N, Suyama H, et al (2001) Nonlinear behavior of human respiratory movement during different sleep stages. Chronobiol Int 18:71-83

21. Poon CS, Barahona M (2001) Titration of chaos with added noise. Proc Natl Acad Sci U S A 98:7107-12

22. Teulier M, Fiamma MN, Straus C, Similowski T (2013) Acute bronchodilation increases ventilatory complexity during resting breathing in stable COPD: toward mathematical biomarkers of ventilatory function? Respir Physiol Neurobiol 185:477-80

23. Mangin L, Clerici C, Similowski T, Poon CS (2009) Chaotic dynamics of cardioventilatory coupling in humans: effects of 
ventilatory modes. American journal of physiology Regulatory, integrative and comparative physiology 296:R1088-1097

24. Schmidt M, Demoule A, Cracco C, et al (2010) Neurally adjusted ventilatory assist increases respiratory variability and complexity in acute respiratory failure. Anesthesiology 112:670-81

25. Briggs K (1990) An improved method for estimating Lyapunov exponents of chaotic time series. Physics Letters 151:27-32

26. Schurmann T, Grassberger P (1996) Entropy estimation of symbol sequences. Chaos 6:414-27

27. El-Khatib M, Jamaleddine G, Soubra R, Muallem M (2001) Pattern of spontaneous breathing: potential marker for weaning outcome. Spontaneous breathing pattern and weaning from mechanical ventilation. Intensive Care Med 27:52-8

28. Mangin L, Fiamma MN, Straus C, et al (2008) Source of human ventilatory chaos: lessons from switching controlled mechanical ventilation to inspiratory pressure support in critically ill patients. Respir Physiol Neurobiol 161:189-96

29. Wrigge H, Golisch W, Zinserling J, et al (1999) Proportional assist versus pressure support ventilation: effects on breathing pattern and respiratory work of patients with chronic obstructive pulmonary disease. Intensive care medicine 25:790-8

30. Fernandez-Vivas M, Caturla-Such J, Gonzalez de la Rosa J, et al (2003) Noninvasive pressure support versus proportional assist ventilation in acute respiratory failure. Intensive Care Med 29:1126-33

31. Gay PC, Hess DR, Hill NS (2001) Noninvasive proportional assist ventilation for acute respiratory insufficiency. Comparison with pressure support ventilation. Am J Respir Crit Care Med 164:1606-11

32. Wysocki M, Richard JC, Meshaka P (2002) Noninvasive proportional assist ventilation compared with noninvasive pressure support ventilation in hypercapnic acute respiratory failure. Critical Care Med 30:323-9

33. Hernandez P, Maltais F, Gursahaney A, et al (2001) Proportional assist ventilation may improve exercise performance in severe chronic obstructive pulmonary disease. J Cardiopulm Rehabil 21:135-42

34. Appendini L, Purro A, Gudjonsdottir M, et al (1999) Physiologic response of ventilator-dependent patients with chronic obstructive pulmonary disease to proportional assist ventilation and continuous positive airway pressure. Am J Respir Crit Care Med 159 (5 Pt 1):1510-7

35. Giannouli E, Webster K, Roberts D, Younes M (1999) Response of ventilator-dependent patients to different levels of pressure support and proportional assist. Am J Respir Crit Care Med 159:1716-25

36. Passam F, Hoing S, Prinianakis G, et al (2003) Effect of different levels of pressure support and proportional assist ventilation on breathing pattern, work of breathing and gas exchange in mechanically ventilated hypercapnic COPD patients with acute respiratory failure. Respiration 70:355-61

37. Xirouchaki N, Kondili E, Vaporidi K, et al (2008) Proportional assist ventilation with load-adjustable gain factors in critically ill patients: comparison with pressure support. Intensive Care Med 34:2026-34

38. Lourenco RV, Cherniack NS, Malm JR, Fishman AP (1966) Nervous output from the respiratory center during obstructed breathing. J Appl Physiol 21:527-33

39. Coisel Y, Chanques G, Jung B, et al (2010) Neurally adjusted ventilatory assist in critically ill postoperative patients: a crossover randomized study. Anesthesiology 113:925-35

40. Patroniti N, Bellani G, Saccavino E, et al (2012) Respiratory pattern during neurally adjusted ventilatory assist in acute respiratory failure patients. Intensive Care Med 38:230-9

41. Suki B, Alencar AM, Sujeer MK, et al (1998) Life-support system benefits from noise. Nature 393:127-8

42. Vassilakopoulos T, Zakynthinos S (2008) When mechanical ventilation mimics nature. Critical Care Med 36:1009-11

43. Carvalho AR, Spieth PM, Guldner A, et al (2011) Distribution of regional lung aeration and perfusion during conventional and noisy pressure support ventilation in experimental lung injury. $\mathrm{J}$ Appl Physiol (1985) 110:1083-92

44. Spieth PM, Guldner A, Beda A, et al (2012) Comparative effects of proportional assist and variable pressure support ventilation on lung function and damage in experimental lung injury. Critical Care Med 40:2654-61

45. Arold SP, Mora R, Lutchen KR, et al (2002) Variable tidal volume ventilation improves lung mechanics and gas exchange in a rodent model of acute lung injury. Am J Respir Crit Care Med 165:366-71

46. Beda A, Spieth PM, Handzsuj T, et al (2010) A novel adaptive control system for noisy pressure-controlled ventilation: a numerical simulation and bench test study. Intensive Care Med 36:164-8

47. Mutch WA, Harms S, Lefevre GR, et al (2000) Biologically variable ventilation increases arterial oxygenation over that seen with positive end-expiratory pressure alone in a porcine model of acute respiratory distress syndrome. Critical Care Med 28:2457-64

48. Mutch WA, Harms S, Ruth Graham M, et al (2000) Biologically variable or naturally noisy mechanical ventilation recruits atelectatic lung. Am J Respir Crit Care Med 162:319-23

49. Mutch WA, Eschun GM, Kowalski SE, et al (2000) Biologically variable ventilation prevents deterioration of gas exchange during prolonged anaesthesia. Br J Anaesth 84:197-203

50. Thammanomai A, Hueser LE, Majumdar A, et al (2008) Design of a new variable-ventilation method optimized for lung recruitment in mice. J Appl Physiol (1985) 104:1329-40

51. Spieth PM, Carvalho AR, Pelosi P, et al (2009) Variable tidal volumes improve lung protective ventilation strategies in experimental lung injury. Am J Respir Crit Care Med 179:684-93

52. Spieth PM, Carvalho AR, Guldner A, et al (2011) Pressure support improves oxygenation and lung protection compared to pressure-controlled ventilation and is further improved by random variation of pressure support. Critical Care Med 39:746-55

53. Arold SP, Suki B, Alencar AM, et al (2003) Variable ventilation induces endogenous surfactant release in normal guinea pigs. Am J Physiol Lung Cell Mol Physiol. 285:L370-375

54. Ma B, Suki B, Bates JH (2011) Effects of recruitment/derecruitment dynamics on the efficacy of variable ventilation. J Appl Physiol (1985) 110:1319-26

55. Felici M, Filoche M, Straus C, et al (2005) Diffusional screening in real 3D human acini--a theoretical study. Respir Physiol Neurobiol 145:279-93

56. Allardet-Servent J (2012) Adding noise to mechanical ventilation: so obvious! Crit Care Med 40:2725-6

57. Bien MY, Hseu SS, Yien HW, et al (2004) Breathing pattern variability: a weaning predictor in postoperative patients recovering from systemic inflammatory response syndrome. Intensive Care Med 30:241-7

58. Bien MY, Shui Lin Y, Shih CH, et al (2011) Comparisons of predictive performance of breathing pattern variability measured during T-piece, automatic tube compensation, and pressure support ventilation for weaning intensive care unit patients from mechanical ventilation. Crit Care Med 39:2253-62

59. Wysocki M, Cracco C, Teixeira A, et al (2006) Reduced breathing variability as a predictor of unsuccessful patient separation from mechanical ventilation. Crit Care Med 34:2076-83

60. Engoren M (1998) Approximate entropy of respiratory rate and tidal volume during weaning from mechanical ventilation. Crit Care Med 26:1817-23

61. Gutierrez G, Das A, Ballarino G, et al (2013) Decreased respiratory rate variability during mechanical ventilation is associated with increased mortality. Intensive Care Med 39:1359-67

62. Bradley BD, Green G, Ramsay T, Seely AJ (2013) Impact of sedation and organ failure on continuous heart and respiratory rate variability monitoring in critically ill patients: a pilot study. Crit Care Med 41:433-44 